\title{
Outcome and prognostic factors of lung cancer patients admitted to the medical intensive care unit
}

\author{
A.K. Adam* and A.O. Soubani ${ }^{\#}$
}

ABSTRACT: The aim of the present study was to assess the outcome of lung cancer patients who were admitted to a medical intensive care unit (MICU) and to identify the measurable predictors of their MICU outcome.

The retrospective study took place at the MICUs of a university-affiliated medical centre and involved adult lung cancer patients admitted to the MICU between January 1998 and October 2005.

A total of 139 lung cancer patients were included during the study period. The mean age \pm SD at MICU admission was $64.2 \pm 10.2$ yrs (48\% males, $52 \%$ females). In total, 96 (69\%) patients had nonsmall cell lung cancer, 18 (13\%) patients had small cell lung cancer, and one patient had mesothelioma. The MICU mortality was $22 \%$ (31 patients), while the in-hospital mortality was $40 \%$ (56 patients). Sixty-eight (49\%) patients required mechanical ventilation (MV), with MICU mortality of $38 \%$ and in-hospital mortality of $53 \%$. The independent predictors of poor MICU outcome were: the need for MV; Acute Physiology And Chronic Health Evaluation III and Simplified Acute Physiology Score III scores; the use of vasopressors; positive blood cultures; high serum lactate; two or more organ system failures; and the need for adult cardiac life support. On multivariate analysis, only the need for vasopressors and the presence of two or more organ system failures predicted poor MICU outcome.

The present study shows that the medical intensive care unit outcome of lung cancer patients is better than previously reported. Intensive care and mechanical ventilation should not be considered futile care in this patient population. While there were no absolute predictors of mortality, the need for vasopressors and the presence of two or more organ system failures predicted poor medical intensive care unit care.

KEYWORDS: Lung cancer, mechanical ventilation, medical intensive care unit, multi-organ system failure, outcome, prognosis

ung cancer is the third most common malignancy but remains the leading cause of cancer mortality in both males and females in the USA and throughout the world. The 1-yr relative survival for lung cancer has increased from $37 \%$ in 1975 to $42 \%$ in 1999-2001, largely due to improvement in surgical technique and combined therapies; however, the 5-yr survival rate for all stages combined is only $15 \%$ [1-5]. Despite this poor prognosis, lung cancer patients are often admitted to the medical intensive care unit (MICU) for critical illness either related to their underlying malignancy or comorbid illnesses, regardless of their cancer cell type or disease stage [6-18].

For editorial comments see page 3 .
Previous studies on cancer patients who were admitted to the MICU [6-21] and, specifically, the few studies on lung cancer patients [22-26], have shown that the outcome of lung cancer patients who were admitted to MICU, especially those requiring mechanical ventilation (MV), is extremely poor. The present study was conducted to assess the outcome of a recent cohort of lung cancer patients admitted to the MICU, including those who required $\mathrm{MV}$, and to identify the measurable predictors of adverse MICU outcomes.

\section{MATERIALS AND METHODS}

The present study was conducted at a Wayne State University-affiliated medical centre (Detroit, MI, USA). The medical centre consists of tertiary-care teaching hospitals and a comprehensive cancer centre. The criteria for admission

\section{AFFILIATIONS}

*Division of Pulmonary, Allergy, Critical Care and Sleep Medicine, and

\#Wayne State University School of Medicine and Karmanos Cancer Center, Detroit, MI, USA.

CORRESPONDENCE

A.O. Soubani

Division of Pulmonary

Allergy

Critical Care and Sleep Medicine 3990 John R- 3 Hudson

Detroit

MI 48201

USA

Fax: 13139930562

E-mail: asoubani@med.wayne.edu

Received:

March 152007

Accepted after revision:

August 062007

STATEMENT OF INTEREST

None declared.
European Respiratory Journal Print ISSN 0903-1936 Online ISSN 1399-3003 
to and discharge from the MICU follow the guidelines set by the American College of Critical Care Medicine and Society of Critical Care Medicine [27]. The MICU is managed by full-time faculty members of the Pulmonary and Critical Care Division. Medical oncologists also conducted daily rounds on oncology patients in the MICU.

After obtaining approval from the institutional review board a retrospective analysis of the medical records of lung cancer patients who were admitted to the MICU between January 1998 and October 20005 was performed. The criterion for including patients in the present study was a histologically proven diagnosis of lung cancer made within the last 2 yrs prior to their admission to the MICU. Patients who stayed in the MICU for $<24 \mathrm{~h}$ and those admitted for routine postoperative care were excluded from the study. For those patients who were admitted more than once to the MICU during the same hospitalisation, only the first MICU admission was analysed.

Demographic, physiological and clinical data including age, sex, race, smoking history, comorbidities, type and stage of lung cancer on admission to the MICU were collected. Attempts were made to determine the indication for admission to the MICU based on clinical and laboratory parameters. In addition, laboratory data obtained within $24 \mathrm{~h}$ of admission to the MICU were collected. If the laboratory values were not available within $24 \mathrm{~h}$ of MICU admission, the values obtained up to $72 \mathrm{~h}$ prior to MICU admission were used. These laboratory data included the following: haemoglobin; white blood cell count; platelet count; coagulation profile; blood urea nitrogen level; creatinine level; electrolyte levels; liver function tests; arterial blood gas measurements; serum lactate level; and blood cultures. Radiological findings were also recorded.

Acute Physiology And Chronic Health Evaluation (APACHE) III score and Simplified Acute Physiology Score (SAPS) III were collected retrospectively for each patient based on data collected within the first $24 \mathrm{~h}$ after their admission to the MICU [28]. The present authors made every attempt to determine the number and type of organ system failure during each patient's MICU stay.

Organ-system failure was recorded if the patient had one or more of the following conditions occur during their MICU stay: respiratory failure (i.e. the presence of hypoxaemia or hypercapnia, or the need for intubation and MV); cardiovascular failure (i.e. the presence of congestive heart failure, the occurrence of ventricular tachycardia or fibrillation, or the need for intravenous infusion of dobutamine, norepinephrine, vasopressin, or epinephrine at any dose, or for dopamine at $>5 \mu \mathrm{g} \cdot \mathrm{kg}^{-1} \cdot \mathrm{min}^{-1}$ for $\geqslant 4 \mathrm{~h}$ ); renal failure (i.e. serum creatinine level $\geqslant 3.4 \mathrm{mg} \cdot \mathrm{dL}^{-1}$, or the need for haemodialysis); neurological failure (i.e. Glasgow coma scale $\leqslant 6$ when available, or subjective criteria, such as the presence of confusion, decreased responsiveness, or coma in the absence of sedation); and hepatic failure (total bilirubin level $\geqslant 4 \mathrm{mg} \cdot \mathrm{dL}^{-1}$ ). Sepsis was defined according the criteria developed by the American College of Chest Physicians and Society of Critical Care Medicine Consensus Conference [19, 29].

MICU data, including indication and duration of MV, noninvasive ventilation and vasopressor use, were also reviewed. If the patient died, the mode of death, such as withdrawal of life-sustaining support or failure to recover spontaneous circulation after adult cardiac life support (ACLS) protocol, was documented.

All patients were evaluated longitudinally to determine their MICU and hospital outcomes. In addition, the 6-month survival rate was also recorded when data were available. Values were reported as the median and/or the mean \pm SD. All percentages were approximated to round numbers. Parametric interval data were initially analysed using a two-tailed t-test. These data are listed as the mean $\pm \mathrm{SD}$. Nominal data were analysed using Chi-squared analysis with Yates' continuity correction or Fisher's exact test when appropriate.

Multiple logistic regression analysis was used to identify the variables that were independently associated with death. Each variable that was found to be significant at $p<0.05$ by univariate analysis was introduced into a backward, stepwise, logistic regression model. A p-value of $<0.05$ was used to indicate statistical significance.

\section{RESULTS}

During the study period, there were 139 lung cancer patients admitted to the MICU. The total number of patients with lung cancer admitted to the hospital during the study period was not available. While data were not available on the number of lung cancer patients who may have had critical illness where the patient, family or treating physicians decided not to transfer to the MICU, it is the general policy and practice at the present authors' institution to transfer oncology patients, including lung cancer patients, to the MICU when they develop critical illness. The baseline clinical characteristics of patients on admission to the MICU are summarised in table 1. Their mean age $\pm S D$ was $64.2 \pm 10.2$ yrs. Of the patients, $48 \%$ were males and $52 \%$ were females. A total of $95(68 \%)$ patients were African-American, which probably reflects the local population demographics. Smoking history was documented in $129(93 \%)$ patients.

Ninety-six (69\%) patients had nonsmall cell lung cancer, 18 $(13 \%)$ patients had small cell lung cancer, one patient had mesothelioma, and in the remaining $24(17 \%)$ patients, the type of lung cancer could not be determined, based on the available medical records. In patients with nonsmall cell lung cancer, 56 patients had stage 4, 28 patients had stage 3 , one patient had stage 2 , and five patients had stage 1 disease. In patients with small cell lung cancer, seven patients had limited disease and eight patients had extensive disease. In 34 (24\%) patients, the stage of lung cancer was not available. The type and stage of lung cancer was unknown in some patients, either because they were diagnosed in other hospitals prior to transfer to the present authors' MICU, or the staging was not completed upon their admission to the MICU.

There were no significant differences in the baseline clinical characteristics between survivors and nonsurvivors during the MICU admission, with the exception of African-American race, history of smoking and nonsmall cell type, which were all associated with favourable outcome (table 1).

The main indications for admission to the MICU are summarised in table 2. Pneumonia was the most common 


\begin{tabular}{|c|c|c|c|c|}
\hline \multirow{2}{*}{$\begin{array}{l}\text { TABLE } 1 \\
\text { Variables }\end{array}$} & \multicolumn{4}{|c|}{$\begin{array}{l}\text { Baseline clinical characteristics of all lung cancer } \\
\text { patients, survivors and nonsurvivors admitted to } \\
\text { the medical intensive care unit }\end{array}$} \\
\hline & All patients & Survivors & Nonsurvivors & -value ${ }^{\#}$ \\
\hline Subjects & 139 & 108 & 31 & \\
\hline Age yrs & $64.2 \pm 10.2$ & $63.6 \pm 10.4$ & $66.5 \pm 9.4$ & 0.15 \\
\hline \multicolumn{5}{|l|}{ Sex } \\
\hline Male & $67(48)$ & $53(49)$ & $14(45)$ & 0.15 \\
\hline Female & $72(52)$ & $55(51)$ & $17(55)$ & 0.15 \\
\hline \multicolumn{5}{|l|}{ Race } \\
\hline White & 41 & 30 & 11 & 0.12 \\
\hline African-American & 95 & 78 & 17 & 0.03 \\
\hline Other & 3 & 0 & 3 & 0.01 \\
\hline Smoking history & $129(93)$ & $103(95)$ & $26(84)$ & 0.04 \\
\hline \multicolumn{5}{|l|}{ Type of lung cancer } \\
\hline Nonsmall cell & $96(69)$ & 79 (73) & $17(55)$ & 0.03 \\
\hline Small & $18(13)$ & $13(12)$ & $5(16)$ & 0.19 \\
\hline Other & 1 & 0 & $1(3)$ & 0.22 \\
\hline Unknown & $24(17)$ & $16(15)$ & $8(26)$ & 0.08 \\
\hline \multicolumn{5}{|c|}{ Stage of lung cancer } \\
\hline 1 & $5(4)$ & $4(4)$ & $1(3)$ & 0.41 \\
\hline 2 & $1(<1)$ & $1(1)$ & 0 & 0.77 \\
\hline 3 & $28(20)$ & $23(21)$ & $5(16)$ & 0.17 \\
\hline 4 & $56(40)$ & $44(41)$ & $12(39)$ & 0.16 \\
\hline Limited disease & 7 (5) & $5(5)$ & $2(6)$ & 0.30 \\
\hline Extensive disease & $8(6)$ & $7(6)$ & $1(3)$ & 0.30 \\
\hline Unknown & $34(24)$ & $24(22)$ & $10(32)$ & 0.09 \\
\hline \multicolumn{5}{|l|}{ Treatment received } \\
\hline Chemotherapy & $8(6)$ & $5(5)$ & $3(10)$ & 0.18 \\
\hline Radiation therapy & $26(19)$ & $23(22)$ & $3(10)$ & 0.08 \\
\hline Surgery & $11(8)$ & $8(7)$ & $3(10)$ & 0.25 \\
\hline Combination & $47(34)$ & $35(32)$ & $12(38)$ & 0.14 \\
\hline None & $43(31)$ & 35 (32) & $8(26)$ & 0.14 \\
\hline Unknown & $4(3)$ & $2(2)$ & $2(6)$ & 0.18 \\
\hline
\end{tabular}

Data are presented as $n$, mean $\pm S D$ or $n(\%)$, unless otherwise indicated. ${ }^{\#}$ : a pvalue $<0.05$ was considered significant.

respiratory indication for MICU admission (26 patients); other respiratory conditions included chronic obstructive pulmonary disease exacerbation, pulmonary oedema, haemoptysis, postbronchoscopy procedures, advanced lung cancer and malignant pleural effusions. The main cardiac indications were arrhythmias (13 patients), cardiac arrest prior to MICU admission, myocardial infarction, congestive heart failure and pericardial effusion. Seizure disorder and mental status changes were equally the most common neurological indications (three patients each), while one patient had stroke. Hyponatraemia was the most common metabolic/electrolytes indication (four patients) for MICU admission.

A total of 68 patients (49\%) required intubation and MV during their stay in the MICU. Table 3 describes the clinical features of the patients who received MV. Of those who underwent MV, $42(62 \%)$ survived their MICU stay and $32(47 \%)$ patients were discharged alive from the hospital. This was in contrast to the outcome of the 71 patients who did not require MV, of whom $66(93 \%)$ patients were discharged alive from the MICU and 51

\begin{tabular}{|c|c|c|c|c|}
\hline \multirow{2}{*}{$\begin{array}{l}\text { TABLE } 2 \\
\text { Indication }\end{array}$} & \multicolumn{4}{|c|}{$\begin{array}{l}\text { Main indication by organ system for medical } \\
\text { intensive care unit admission for all lung cancer } \\
\text { patients, survivors and nonsurvivors }\end{array}$} \\
\hline & All patients & Survivors & Nonsurvivors & p-value $\#$ \\
\hline Subjects & 139 & 108 & 31 & \\
\hline Respiratory & $68(49)$ & $50(46)$ & $18(58)$ & 0.08 \\
\hline Cardiovascular & $34(25)$ & $29(27)$ & $5(16)$ & 0.09 \\
\hline Sepsis & $11(8)$ & $5(5)$ & $6(20)$ & 0.01 \\
\hline Neurological & 7 (5) & $7(6)$ & 0 & 0.16 \\
\hline Renal & $2(1)$ & $1(1)$ & $1(3)$ & 0.35 \\
\hline Metabolic & $9(7)$ & $8(7)$ & $1(3)$ & 0.27 \\
\hline Bleeding & $2(1)$ & $2(2)$ & 0 & 0.60 \\
\hline Others & $6(4)$ & $6(6)$ & 0 & 0.21 \\
\hline
\end{tabular}

Data are presented as $\mathrm{n}$ or $\mathrm{n}(\%)$, unless otherwise indicated. ${ }^{*}$ : a $\mathrm{p}$-value $<0.05$ was considered significant.

(72\%) were discharged alive from the hospital. There was no significant difference in the duration of MV between the survivor and nonsurvivor groups $(p=0.18)$. The most common indications for MV were pulmonary/airway problems in 46 $(68 \%)$ patients, cardiovascular problems in $12(18 \%)$ patients and sepsis in seven $(10 \%)$ patients. Pulmonary indications for MV were associated with favourable MICU outcome $(p=0.04)$, while those with sepsis as the main indication for MV had poor MICU outcome $(p=0.001)$. Those mechanically ventilated patients with high APACHE III and SAPS III scores on admission to the MICU and those with high serum lactate had an unfavourable outcome.

The median length of stay in the MICU was 2 days (range 131 days), and this was similar in survivors and nonsurvivors. Thirty-one (22\%) patients died during their MICU stay and 56 (40\%) patients died during their hospitalisation. During their MICU stay, the decision was made to forego life-sustaining care in $44(32 \%)$ patients. Of these, 21 died in the MICU. ACLS was performed on 10 patients and only one patient survived the MICU stay, but subsequently died in the hospital.

Acuity scores, MICU data and main laboratory variables on admission to the MICU were recorded and compared between survivors and nonsurvivors, as shown in table 4 . The initial APACHE III and SAPS III scores demonstrated significant differences between survivors and nonsurvivors (mean APACHE III: survivors $54.3 \pm 21.4$, nonsurvivors $85.8 \pm 28.5$, $\mathrm{p}<0.0001$; mean SAPS III: survivors $37.4 \pm 19$, nonsurvivors $66.8 \pm 27.1, \mathrm{p}<0.0001)$. There were significant differences between the two groups regarding the need for vasopressors $(\mathrm{p}<0.0001)$ and MV $(\mathrm{p}<0.0001)$. Other MICU admission laboratory data that were significantly different between the two groups were serum lactate levels, serum calcium levels and positive blood culture results. The serum lactate levels on admission to the MICU were significantly higher in patients who eventually died in the MICU (survivors $1.4 \pm 1.8 \mathrm{mmol} \cdot \mathrm{L}^{-1}$, nonsurvivors $\left.3.7 \pm 4.4 \mathrm{mmol} \cdot \mathrm{L}^{-1}, \mathrm{p}=0.008\right)$. Sixteen $(12 \%)$ patients had positive blood culture results either during the $24 \mathrm{~h}$ immediately prior to MICU admission or within the first $48 \mathrm{~h}$ after MICU admission. The most common 


\begin{tabular}{|c|c|c|c|c|c|}
\hline \multirow{2}{*}{$\begin{array}{l}\text { TABLE } 3 \\
\text { Variables }\end{array}$} & \multicolumn{5}{|c|}{$\begin{array}{l}\text { Clinical characteristics of lung cancer patients } \\
\text { requiring mechanical ventilation }\end{array}$} \\
\hline & & All patients & Survivors & Nonsurvivors & p-value ${ }^{\#}$ \\
\hline \multicolumn{2}{|l|}{ Subjects } & 68 & 42 & 26 & \\
\hline \multicolumn{2}{|l|}{ Age yrs } & $65.3 \pm 9.9$ & $65 \pm 10.1$ & $65.8 \pm 9.6$ & 0.74 \\
\hline \multicolumn{6}{|c|}{ Type of lung cancer } \\
\hline \multicolumn{2}{|c|}{ Nonsmall cell } & $42(62)$ & $29(69)$ & $13(50)$ & 0.06 \\
\hline \multicolumn{2}{|l|}{ Small } & $9(13)$ & $4(10)$ & $5(19)$ & 0.15 \\
\hline \multicolumn{2}{|l|}{ Other } & $1(1)$ & 0 & $1(4)$ & 0.38 \\
\hline \multicolumn{2}{|l|}{ Unknown } & $16(24)$ & 9 (21) & $7(27)$ & 0.20 \\
\hline \multicolumn{6}{|c|}{ Stage of lung cancer } \\
\hline \multicolumn{2}{|l|}{1} & 4 & 3 & 1 & 0.37 \\
\hline \multicolumn{2}{|l|}{2} & 0 & 0 & 0 & \\
\hline \multicolumn{2}{|l|}{3} & 13 & 8 & 5 & 0.25 \\
\hline \multicolumn{2}{|l|}{4} & 24 & 15 & 9 & 0.21 \\
\hline \multicolumn{2}{|c|}{ Limited disease } & 6 & 4 & 2 & 0.33 \\
\hline \multicolumn{2}{|c|}{ Extensive disease } & 1 & 0 & 1 & 0.38 \\
\hline \multicolumn{2}{|l|}{ Unknown } & 20 & 12 & 8 & 0.21 \\
\hline \multicolumn{6}{|c|}{ Indication for MV } \\
\hline \multicolumn{2}{|c|}{ Pulmonary/airway } & $46(68)$ & $32(76)$ & $14(54)$ & 0.04 \\
\hline \multicolumn{2}{|c|}{ Cardiovascular } & $12(18)$ & $7(17)$ & $5(19)$ & 0.24 \\
\hline \multicolumn{2}{|c|}{ Sepsis } & $7(10)$ & $1(2)$ & $6(23)$ & 0.001 \\
\hline \multicolumn{2}{|l|}{ Others } & $3(4)$ & $2(5)$ & $1(4)$ & 0.45 \\
\hline \multicolumn{2}{|l|}{ SAPS III } & $52 \pm 26.6$ & $42 \pm 20.4$ & $67.1 \pm 29$ & 0.0006 \\
\hline \multicolumn{2}{|c|}{ APACHE III score } & $69.4 \pm 28.3$ & $59.6 \pm 22.2$ & $85 \pm 30.3$ & 0.0006 \\
\hline \multicolumn{2}{|l|}{$\begin{array}{l}\text { Lactate level } \\
\mathrm{mmol} \cdot \mathrm{L}^{-1}\end{array}$} & $2.86 \pm 3.4$ & $2 \pm 1.97$ & $4.2 \pm 4.6$ & 0.03 \\
\hline \multicolumn{2}{|c|}{ MV duration $>24 h$} & 47 (69) & $30(71)$ & $17(65)$ & 0.18 \\
\hline
\end{tabular}

Data are presented as $n$, mean $\pm S D$ or $n(\%)$, unless otherwise indicated. MV: mechanical ventilation; SAPS: Simplified Acute Physiology Score; APACHE: Acute Physiology And Chronic Health Evaluation. ${ }^{\#}$ : a p-value $<0.05$ was considered significant.

isolates were Gram-positive cocci in 14 patients and Gramnegative bacilli in three patients. One patient had two organisms isolated from blood culture. Patients with positive blood cultures had a poor outcome $(p=0.001)$. No differences in outcome were observed based on the type of organism isolated. There was no significant difference between survivors and nonsurvivors regarding the type of lung cancer treatment, whether it was surgery $(p=0.25)$, chemotherapy $(p=0.18)$, radiation therapy $(p=0.08)$ or combination therapy $(p=0.14)$.

According to the criteria described in the Materials and methods section, $115(83 \%)$ patients had one or more organ system failure. Mortality increased with the number of organ systems that failed beyond one organ system failure, and this trend was seen with all types of organ system failure.

To determine the predictors of MICU outcomes, statistically significant MICU data, physiological and laboratory variables on MICU (table 4), as well as the need for MV, the use of vasopressors and the presence of multiorgan system failures (MOSFs), were entered into a stepwise backward elimination regression analysis model. Race and smoking history were not included in the regression analysis. Only the need for vasopressors and the presence of two or more MOSFs during
TABLE 4 Acuity scores, vasopressors, mechanical ventilation (MV) and main laboratory variables on medical intensive care unit admission for survivors and nonsurvivors

\begin{tabular}{|c|c|c|c|}
\hline Variables & Survivors & Nonsurvivors & p-value ${ }^{\#}$ \\
\hline Subjects & 108 & 31 & \\
\hline SAPS III & $37.4 \pm 19$ & $66.8 \pm 27.1$ & $<0.0001$ \\
\hline APACHE III score & $54.3 \pm 21.4$ & $85.8 \pm 28.5$ & $<0.0001$ \\
\hline Vasopressors use & 12 & 23 & $<0.0001$ \\
\hline MV & 42 & 26 & $<0.0001$ \\
\hline Lactate $\mathrm{mmol} \cdot \mathrm{L}^{-1}$ & $1.4 \pm 1.8$ & $3.7 \pm 4.4$ & 0.008 \\
\hline Albumin gm $\cdot \mathrm{dL}^{-1}$ & $1.7 \pm 1.4$ & $1.3 \pm 1.2$ & 0.12 \\
\hline Calcium $\mathrm{mg} \cdot \mathrm{dL}^{-1}$ & $8.7 \pm 1.8$ & $7.4 \pm 2.2$ & 0.005 \\
\hline Sodium $\mathrm{mmol} \cdot \mathrm{L}^{-1}$ & $137.2 \pm 8.2$ & $134.6 \pm 27$ & 0.60 \\
\hline Creatinine $\mathrm{mg} \cdot \mathrm{dL}^{-1}$ & $1.6 \pm 1.9$ & $1.4 \pm 0.9$ & 0.41 \\
\hline $\mathrm{T}$. bilirubin $\mathrm{mg} \cdot \mathrm{dL}^{-1}$ & $0.4 \pm 0.5$ & $0.4 \pm 0.8$ & 0.99 \\
\hline Haematocrit \% & $33.2 \pm 6.7$ & $31.2 \pm 5.4$ & 0.09 \\
\hline Platelets $1000 \times \mathrm{mm}^{3}$ & $289.4 \pm 130$ & $259.8 \pm 139.8$ & 0.29 \\
\hline Positive blood culture & 7 & 9 & 0.001 \\
\hline
\end{tabular}

Data are presented as $n$ or mean $\pm S D$, unless otherwise indicated. SAPS: Simplified Acute Physiology Score; APACHE: Acute Physiology And Chronic Health Evaluation. ${ }^{\# \text { : a }}$-value $<0.05$ was considered significant.

their MICU stay predicted poor MICU outcome (table 5). The final model showed good discrimination and calibration.

\section{DISCUSSION}

The aim of the present study was to assess the outcome of lung cancer patients who had been admitted to the present authors' MICU and to identify the predictors of MICU adverse outcome for this group. The data showed that the MICU mortality rate was $22 \%$, while the in-hospital mortality was $40 \%$. The predicted MICU mortality for these patients based on the APACHE III score was 29\%. For those who required MV (49\% of the patients), the MICU mortality was $38 \%$ and the inhospital mortality was $53 \%$. The independent predictors of adverse MICU outcome in this patient population were haemodynamic instability requiring vasopressor use and the presence of MOSF.

The present study shows that the outcome of lung cancer patients admitted to the MICU has improved significantly compared with historical studies and confirms the findings of a recent study on a similar group of patients from Brazil and France (table 6) [22-26, 30]. Furthermore, the present study shows that the mortality rate for those lung cancer patients who require MV is higher than that for the group as a whole; however, the MICU outcome of these patients showed a similar favourable trend compared with previous studies. In the study by LIN et al. [24], on the outcome of lung cancer patients with acute respiratory failure requiring $\mathrm{MV}$, the MICU mortality was $73 \%$ and the in-hospital mortality was $85 \%$. Another study by EWER et al. [25] reported the in-hospital mortality to be as high as $91 \%$ and a 6-month mortality of $98 \%$. In the recent study by SOARES et al. [30], the MICU mortality rate for lung cancer patients who required MV was $56 \%$ and the in-hospital mortality was $69 \%$. 


\begin{tabular}{|c|c|c|c|c|}
\hline \multirow{2}{*}{$\begin{array}{l}\text { TABLE } 5 \\
\text { Variables }\end{array}$} & \multicolumn{4}{|c|}{$\begin{array}{l}\text { Predictors of medical intensive care unit mortality } \\
\text { on stepwise backward elimination regression } \\
\text { analysis }\end{array}$} \\
\hline & & $\begin{array}{l}\text { Odds } \\
\text { ratio }\end{array}$ & $\begin{array}{c}\text { Confidence } \\
\text { interval }\end{array}$ & p-value ${ }^{\#}$ \\
\hline \multicolumn{2}{|c|}{ Vasopressors use } & 8.7 & $2.8-27$ & $<0.0001$ \\
\hline \multicolumn{2}{|c|}{ Multiorgan system failure $\geqslant 2$} & 40.8 & $5.1-328.3$ & $<0.0001$ \\
\hline
\end{tabular}

The improved outcome of lung cancer patients admitted to the MICU reported in the present study and by SOARES et al. [30] may be related to: improved MV strategies that minimise further lung injury; better management of sepsis; a multidisciplinary approach to the management of these cases; and improvement in the therapeutic options for patients with lung cancer. The present study was not designed to test these theories. It is also possible that there is selection bias that resulted in admitting patients who are thought to benefit from intensive care unit care. It is difficult to measure the role of the latter possibility on the improved outcome of lung cancer patients admitted to the MICU. Prospective studies and more strict inclusion criteria are necessary to confirm and address the reasons for the improved survival noticed in these recent studies.

Several studies have tried to identify the clinical variables that are associated with poor MICU outcome. The outcome predictors reported by REICHNER et al. [22] were the need for $\mathrm{MV}$, advanced lung cancer stage and higher sequential organ failure assessment score. In the study by BoussAT et al. [23], acute pulmonary disease and Karnofsky performance status $<70$ were associated with higher mortality. In the recent study by SOARES et al. [30], the predictors of poor MICU outcome were the severity of comorbid illnesses, the number of organ system failures, cancer recurrence or progression, and airway infiltration or obstruction by cancer. In the present study, it was possible to identify several predictors of poor MICU outcome that included high admission APACHE III and SAPS III scores, the need for MV, the use of vasopressors, positive blood cultures, high serum lactate, the presence of two or more organ system failures, and the need for ACLS protocol for cardiopulmonary arrest. However, on multivariate logistic regression, only the use of vasopressors and the presence of two or more MOSF predicted poor MICU outcome, with odds ratios of 8.7 and 40.8 , respectively (table 5). The stage of lung cancer, the presence of metastasis, or the type of cancer therapy did not correlate with poor MICU outcome. This was similar to the findings in the study by Boussat et al. [23] but was in contrast to the findings of REICHNER et al. [22] and SOARES et al. [30]. It was also observed that patients with nonsmall cell lung cancer had a favourable MICU outcome $(\mathrm{p}=0.027)$, which is contrary to the findings of other studies $[22,23]$.

In the present study, only one patient underwent ACLS protocol and survived their MICU stay, but subsequently died

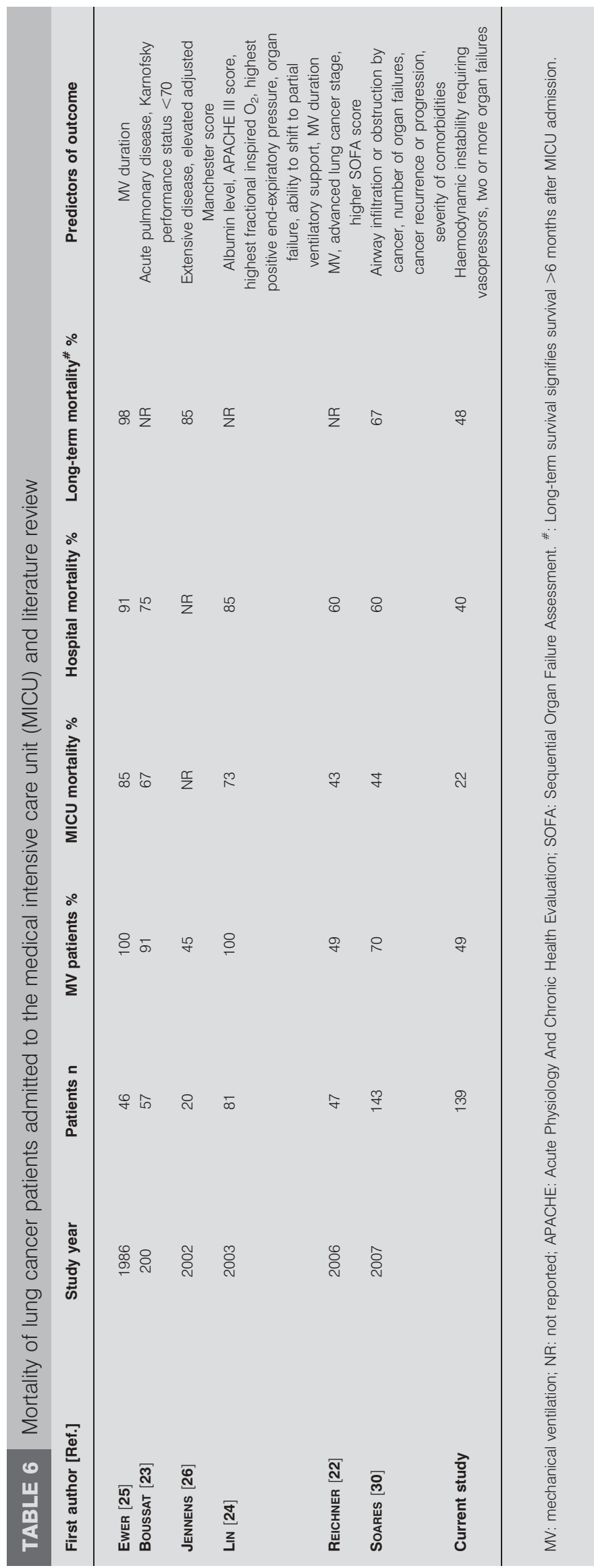

VOLUME 31 NUMBER 1 
in the hospital. Although the number is small, this observation suggests that, while aggressive therapy is appropriate for this patient population, subjecting them to the ACLS protocol appears to be futile, and such an intervention probably should be avoided. This goal could be achieved by addressing the code status and initiating end-of-life discussion early during the course of their illness, probably before the patient's condition deteriorates.

The present study has important limitations, including the retrospective nature of the analysis that may have resulted in selection bias, and the lack of assessment of potentially significant predictors of outcome, such as the role of progression of lung cancer, airway infiltration, the severity of comorbid illnesses, complications of cancer therapy, or performance status in predicting the MICU outcome of lung cancer patients. Prospective, multicentre trials are necessary to address these issues.

In conclusion, the present data have shown that the medical intensive care unit outcome of lung cancer patients is improving and is comparable to other critically ill patient populations. Intensive care and mechanical ventilation should not be regarded as futile care. While there were no absolute predictors of mortality, haemodynamic instability requiring vasopressors use, and the development of two or more organ system failures are less likely to survive their medical intensive care unit care.

\section{REFERENCES}

1 American Cancer Society. Cancer facts and figures 2006. Atlanta, American Cancer Society, 2006.

2 Jemal A, Siegel R, Ward E, et al. Cancer Statistics, 2006. CA Cancer J Clin 2006; 56: 106-130.

3 Jemal A, Chu KC, Tarone RE. Recent trends in lung cancer mortality in the United States. Cancer surveillance series. J Natl Cancer Inst 2001; 93: 277-283.

4 Jemal A, Travis WD, Tarone RE, et al. Lung cancer rates convergence in young men and women in the United States: analysis by birth cohort and histologic type. Int $J$ Cancer 2003; 105: 101-107.

5 Thun MJ, Henley SJ, Burns D, et al. Lung cancer death in lifelong nonsmoker. J Natl Cancer Inst 2006; 98: 691-699.

6 Groeger JS, White P, Nierman DM, et al. Outcome for cancer patients requiring mechanical ventilation. J Clin Oncol 1999; 17: 991-997.

7 Staudinger T, Stoiser B, Mullner M, et al. Outcome and prognostic factors in critically ill cancer patients admitted to the intensive care unit. Crit Care Med 2000; 28: 1322-1328.

8 Thiery G, Azoulay E, Darmon M, et al. Outcome of cancer patients considered for intensive care unit admission: a hospital-wide prospective study. J Clin Oncol 2005; 23: 4406-4413.

9 Kress JP, Christenson J, Pohlman AS, et al. Outcome of critically ill cancer patients in a university hospital setting. Am J Respir Crit Care Med 1999; 160: 1957-1961.

10 Groeger JS, Lemeshow S, Price K, et al. Multicenter outcome study of cancer patients admitted to the intensive care unit: a probability of mortality model. J Clin Oncol 1998; 16: 761-770.
11 Azoulay E, Moreau D, Alberti C, et al. Predictors of shortterm mortality in critically ill patients with solid malignancies. Intensive Care Med 2000; 26: 1817-1823.

12 Ghosen M, Kanso C, Kattan J, et al. Outcome of cancer patients admitted to the intensive care unit (ICU). J Med Liban 2002; 50: 132-136.

13 Groeger JS, Glassman J, Nierman DM, et al. Probability of mortality of critically ill cancer patients at $72 \mathrm{~h}$ of intensive care unit (ICU) management. Support Care Cancer 2003; 11: 686-695.

14 Hauser MJ, Tabak J, Baier H. Survival of patients with cancer in a medical critical care unit. Arch Intern Med 1982; 142: 527-529.

15 Schapira DV, Studnicki J, Bradham DD, et al. Intensive care, survival and expense of treating critically ill cancer patients. JAMA 1993; 269: 783-786.

16 Maschmeyer G, Bertschat FL, Moesta KT, et al. Outcome analysis of 189 consecutive cancer patients referred to the intensive care unit as emergencies during a 2-year period. Eur J Cancer 2003; 39: 783-792.

17 Kongsgaard UE, Meidell NK. Mechanical ventilation in critically ill cancer patients: outcome and utilization of resources. Support Care Cancer 1999; 7: 95-99.

18 Benoit DD, Vandewoude KH, Decruyenaere JM, et al. Outcome and early prognostic indicators in patients with a hematologic malignancy admitted to the intensive care unit for a life-threatening complication. Crit Care Med 2003; 31: 104-112.

19 Soubani AO, Kseibi E, Bander JJ, et al. Outcome and prognostic factors of hematopoietic stem cell transplantation recipients admitted to a medical ICU. Chest 2004; 126 : 1604-1611.

20 Azoulay E, Alberti C, Bornstain C, et al. Improved survival in cancer patients requiring mechanical ventilatory support: impact of noninvasive mechanical ventilatory support. Crit Care Med 2001; 29: 519-525.

21 Azoulay E, Thiery G, Chevret S, et al. The prognosis of acute respiratory failure in critically ill cancer patients. Medicine (Baltimore) 2004; 83: 360-370.

22 Reichner CA, Thompson JA, O'Brien S, et al. Outcome and code status of lung cancer patients admitted to the medical ICU. Chest 2006; 130: 719-723.

23 Boussat S, El'rini T, Dubiez A, et al. Predictive factors of death in primary lung cancer patients on admission to the intensive care unit. Intensive Care Med 2000; 26: 1811-1816.

24 Lin YC, Tsai YH, Huang CC, et al. Outcome of lung cancer patients with acute respiratory failure requiring mechanical ventilation. Respir Med 2004; 98: 43-51.

25 Ewer MS, Ali MK, Atta MS, et al. Outcome of lung cancer patients requiring mechanical ventilation for pulmonary failure. JAMA 1986; 256: 3364-3366.

26 Jennens RR, Rosenthal MA, Mitchell P, Presneill JJ. Outcome of patients admitted to the intensive care unit with newly diagnosed small cell lung cancer. Lung Cancer 2002; 38: 291-296.

27 Task force of the American College of Critical Care Medicine, Society of Critical Care Medicine. Guidelines for intensive care unit admission, discharge and triage. Crit Care Med 1999; 27: 633-638. 
28 Knaus WA, Wagner DP, Draper EA, et al. The APACHE III prognostic system. Risk prediction of hospital mortality for critically ill hospitalized adults. Chest 1991; 100: 1619-1636.

29 Bone RC, Balk RA, Cerra FB, et al. Definitions for sepsis and organ failure and guidelines for the use of innovative therapies in sepsis: the ACCP/SCCM Consensus
Conference Committee; American College of Chest Physicians/Society of Critical Care Medicine. Chest 1992; 101: 1644-1655.

30 Soares M, Darmon M, Salluh JIF, et al. Prognosis of lung cancer patients with life-threatening complications. Chest 2007; 13: 840-846. 